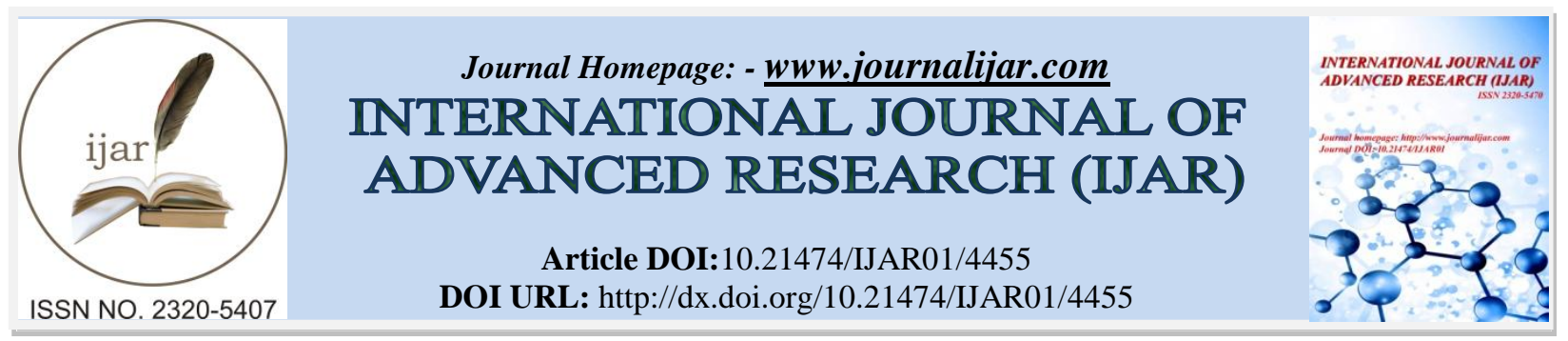

RESEARCH ARTICLE

\title{
INCIDENTAL FINDING OF MICROFILARIAE IN BONE MARROW ASPIRATE OF A PATIENT WITH TUBERCULOSIS: A CASE REPORT.
}

"Somaya Albhaisi M.B.B.ch, Nihal Bashir MD and Tehmina Khan MD.

\section{Manuscript Info}

Manuscript History

Received: 12 April 2017

Final Accepted: 14 May 2017

Published: June 2017

Key words:-

Tuberculosis - Filariasis - Co-infection

- Bone marrow

\section{Abstract}

Filariasis is one of the most prevalent helminthic infections worldwide. Filarial infections are a huge health burden in endemic areas. More than 90 percent of these infections are due to Wuchereria bancrofti.Data recently published by the World Health Organization (WHO) shows that more than 556 million people worldwide were treated for lymphatic Filariasis in 2015. The common features are asymptomatic microfilariaemia, acute adenolymphangitis, hydrocele and chronic lymphatic disease. The risk of developing neurological manifestations may increase in cases that harbor multiple filariasis or coinfections, for instance as with Plasmodium. Microfilariae are an uncommon finding in the bone marrow and their presence may be an incidental finding in the absence of the clinical features of lymphatic filariasis. The peripheral blood may or may not reveal the microfilariae and eosinophilia is absent in a majority of the cases. All the bone marrow aspirates must be screened for microfilariae in the endemic areas, to identify the asymptomatic carriers. We describe a case of TB who was incidentally found to have Filariasis demonstrated in bone marrow aspirate.

\section{Introduction:-}

Lymphatic filariasis is a major public health problem in the endemic areas of India. In the natural history of lymphatic filariasis, the adult worm lodges in the lymphatics and the microfilariae circulate in the blood stream. Lymphatic filariasis commonly presents as lymphangitis, elephantiasis and peripheral blood eosinophilia. During their circulation in the peripheral blood, the microfilariae may get lodged in various organs and body fluids and be picked up in the cytological samples. They are found in the fine needle aspirate smears from the thyroid (1-3), breast (1-3), lymph nodes (1),(3)and subcutaneous nodules (1),(3), in cervical scrape smears (1), in bronchial washings (1),(4) and in body fluids (1). It is also possible that they may get trapped in the bone marrow during their circulation in the peripheral blood. Including the present case, none of the published cases where microfilaria was found in the bone marrow aspirates, had a classical clinical presentation of lymphatic filariasis (5-11). A diagnosis of filariasis was made after demonstrating the microfilariae in the bone marrow aspirate or in the peripheral blood smears. In a few reported cases, microfilariae were present in the bone marrow aspirates but not in the peripheral blood. Microfilariae in the bone marrow aspirates is a rare finding. A few cases have been reported in the literature in association with various hematological findings, such as peripheral blood cytopenia, bone marrow hypoplasia, megaloblastic anemia and acute lymphoid leukemia (5-10). Our patient did not have any lymphatic or cutaneous stigmata of filariasis. The effect of filarial infections on TB progression and/or outcome is still unknown. There is a 
lack of suitable animal models or large human studies of mycobacteria/filaria or malaria/ filaria co-infections to definitively ascertain the interplay between filaria and malaria or TB (11).

\section{Case Presentation:-}

A 27 years old Indian male new in UAE, presented to the Emergency Department (ED) with headache, low back pain and subjective feeling of fever and chills for 4 days, associated with repeated vomiting on the same day of presentation. He came from India one month ago in order to work in a laundry. Shortly after his arrival, he had Tuberculosis (TB) screening for visa purposes which included sputum acid fast bacilli smears and cultures and chest $\mathrm{X}$-ray showed diffuse micro nodules are noted bilaterally with Reticulonodular changes noted in the apices.

He complained of headaches associated with neck pain which got aggravated by coughing.

No known relieving factors. He denied visual disturbances, dizziness, photo phobia, seizures, sore throat, urinary symptoms and gastrointestinal symptoms.

He reported 2 kilograms weight loss over a period of 3 months and had a sick contact with TB patient.

During assessment in the ED, the patient was afebrile hemodynamically stable. On examination, he was conscious, alert and oriented to time place and person, with neck rigidity and negative kerning's and Brudsinski's signs, other systemic examination was unremarkable.

Blood tests results were: White Blood Cell (WBC): $9.2 * 10^{9} / \mathrm{L}$, C-reactive protein (CRP): $20 \mathrm{ng} / \mathrm{mL}$, normal urea electrolytes. Lumbar puncture was performed in the ED. Cerebrospinal fluid (CSF) was hazy, its analysis showed: nucleated cells: $71 * 10^{6} / \mathrm{L}$ with lymphocyte predominance, $84 \%$. Red Blood Cells: $418^{*} 10^{6} / \mathrm{L}$, CSF Glucose 2 $\mathrm{mmol} / \mathrm{L}$ (serum glucose was $4.5 \mathrm{mmol} / \mathrm{L}$ and serum to CSF glucose is 2.25) and CSF protein of $1.58 \mathrm{~g} / \mathrm{L}$. Malaria test was negative and Liver function test were normal. Herpes simplex virus (HSV) Polymerase chain reaction (PCR) for CSF was negative as well as Mycobacterium Tuberculosis PCR. Computed Tomography (CT) brain was unremarkable, Chest x-ray showed features of Miliary TB (image1). The patient was started on vancomycin and ceftriaxone empirically. The patient was started on Isoniazid 300mg, rifampin 600mg, ethambutol 1200mg and pyrazinamide $1500 \mathrm{mg}$ oral tablets.

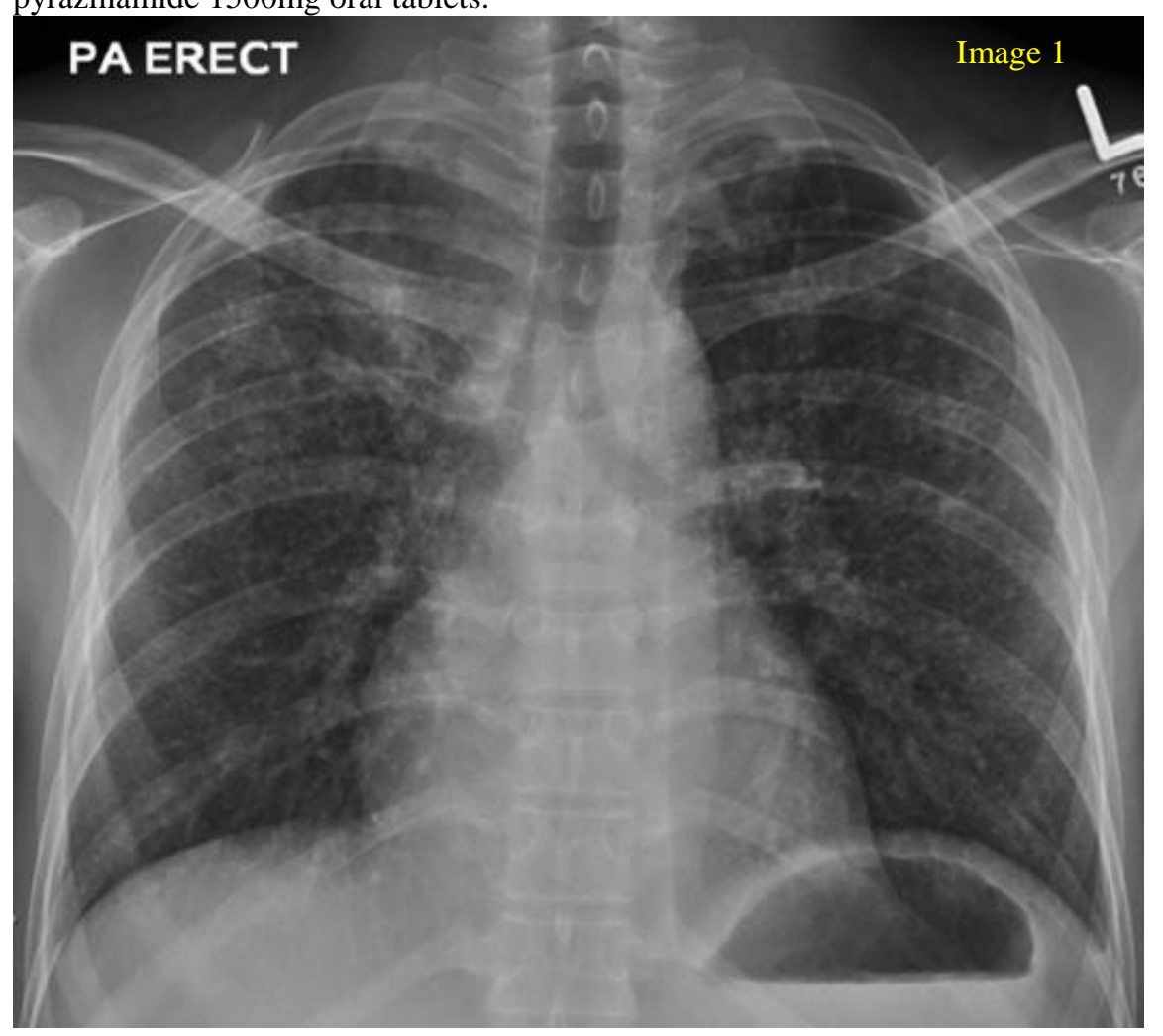


During his third day of hospital stay, the patient developed acute agitation, confusion and focal neurological deficit. He was found to have hyponatremia (serum Sodium was $118 \mathrm{mmol} / \mathrm{L}$ ); however, it was normal on admission. The patient was started on hypertonic saline $2 \%$. Sodium level became normal after 2 days of fluid restriction and hypertonic saline infusion. The patient improved clinically and was discharged home. He presented the next day with frontal headache and photophobia associated with fever. Physical examination was unremarkable. Significant blood tests results were: serum WBC: $21 * 10^{9} / \mathrm{L}$ with neutrophilia, platelets count $444 * 10^{9} / \mathrm{L}, \mathrm{CRP} 6 \mathrm{ng} / \mathrm{mL}$. Lumbar puncture was repeated and CSF analysis showed nucleated cell of $1213 * 10^{6} / \mathrm{L}$ with neutrophilic predominance, protein $1.57 \mathrm{~g} / \mathrm{L}$ and glucose of $1 \mathrm{mmol} / \mathrm{L}$. The patient was started on intravenous ceftriaxone, vancomycin and dexamethasone. CSF was negative for TB PCR. CSF cultures (mycobacterial, bacterial and fungal) were negative. 2 Sputum Acid Fast Bacilli Smears were negative. Cytomegalovirus (CMV) and Epstein-Barr virus (EBV) PCR testing was negative Human immunodeficiency virus (HIV) and toxoplasmosis screen were negative as well. Brain magnetic resonance imaging (MRI) showed numerous miliary round ring enhancing lesions suggestive of Miliary TB [Images 2, 3].

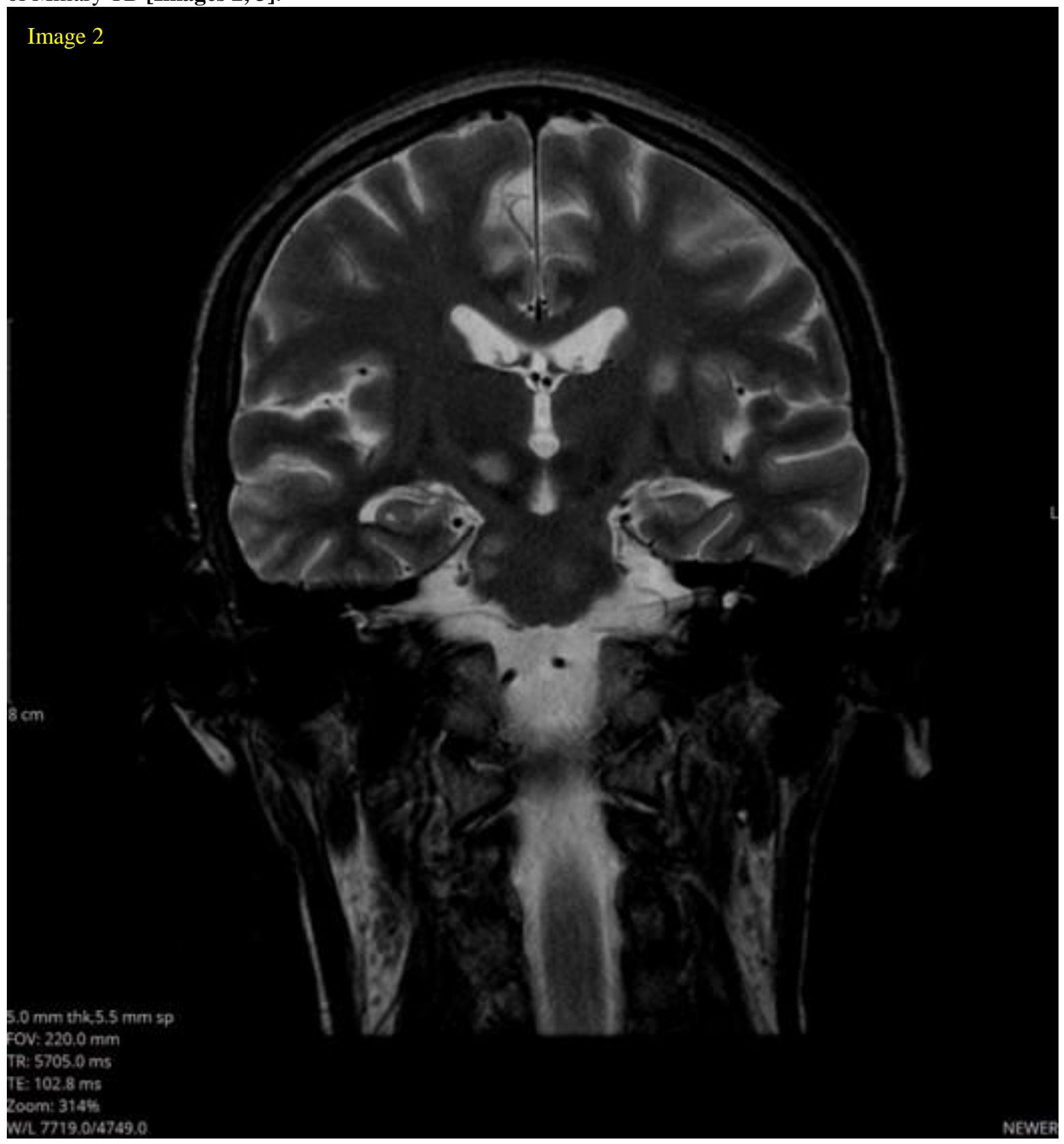




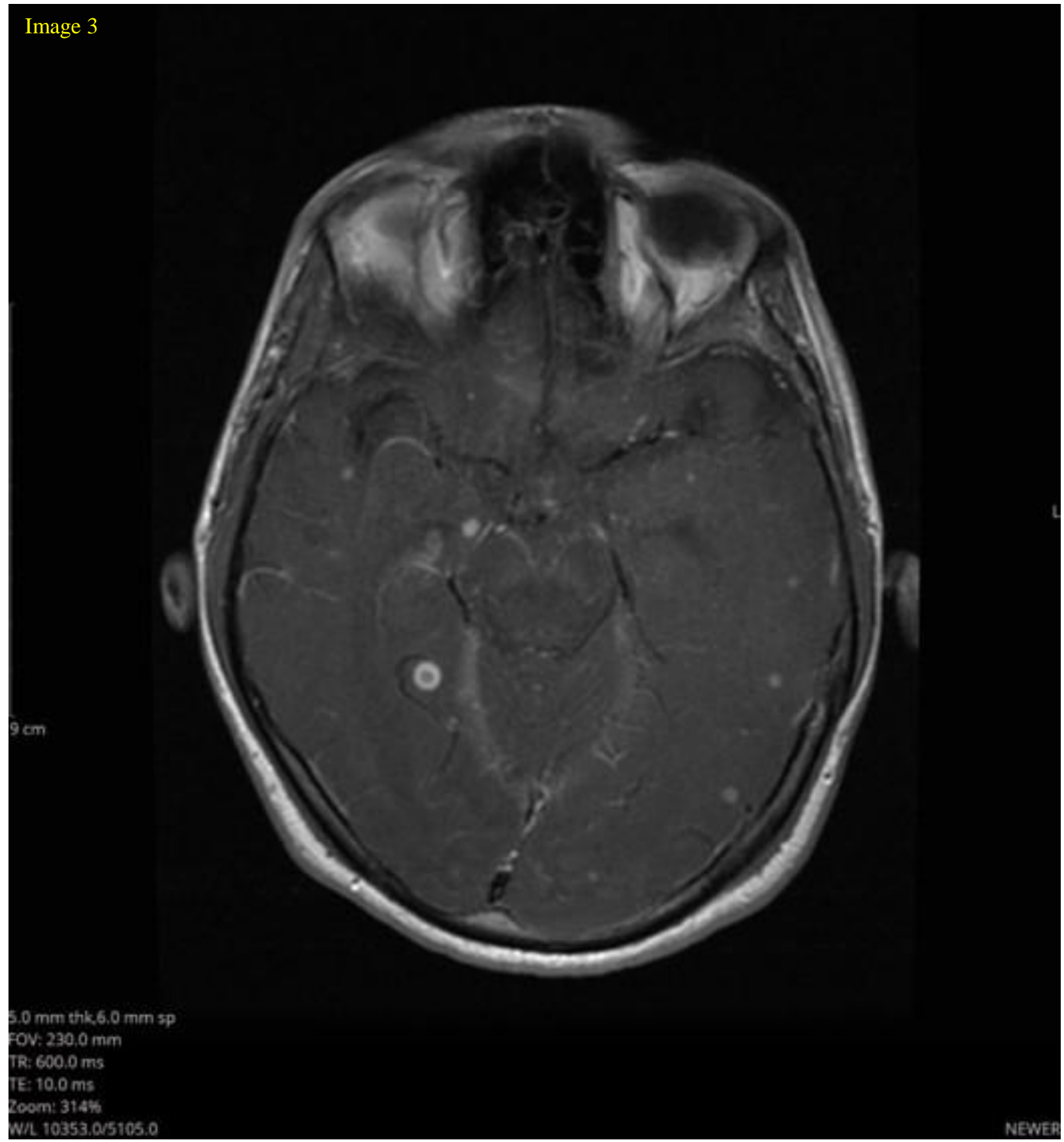

Two final acid fast bacilli sputum cultures were positive and pan sensitive to first line anti-TB medications. Patient underwent bone marrow aspiration which was positive for filariasis[Images 4, 5].

The patient was discharged from the hospital on Anti-TB medications with instructions to be taken for 9 months, in addition to anti-filarial medicine which was Diethylcarbamazine. 


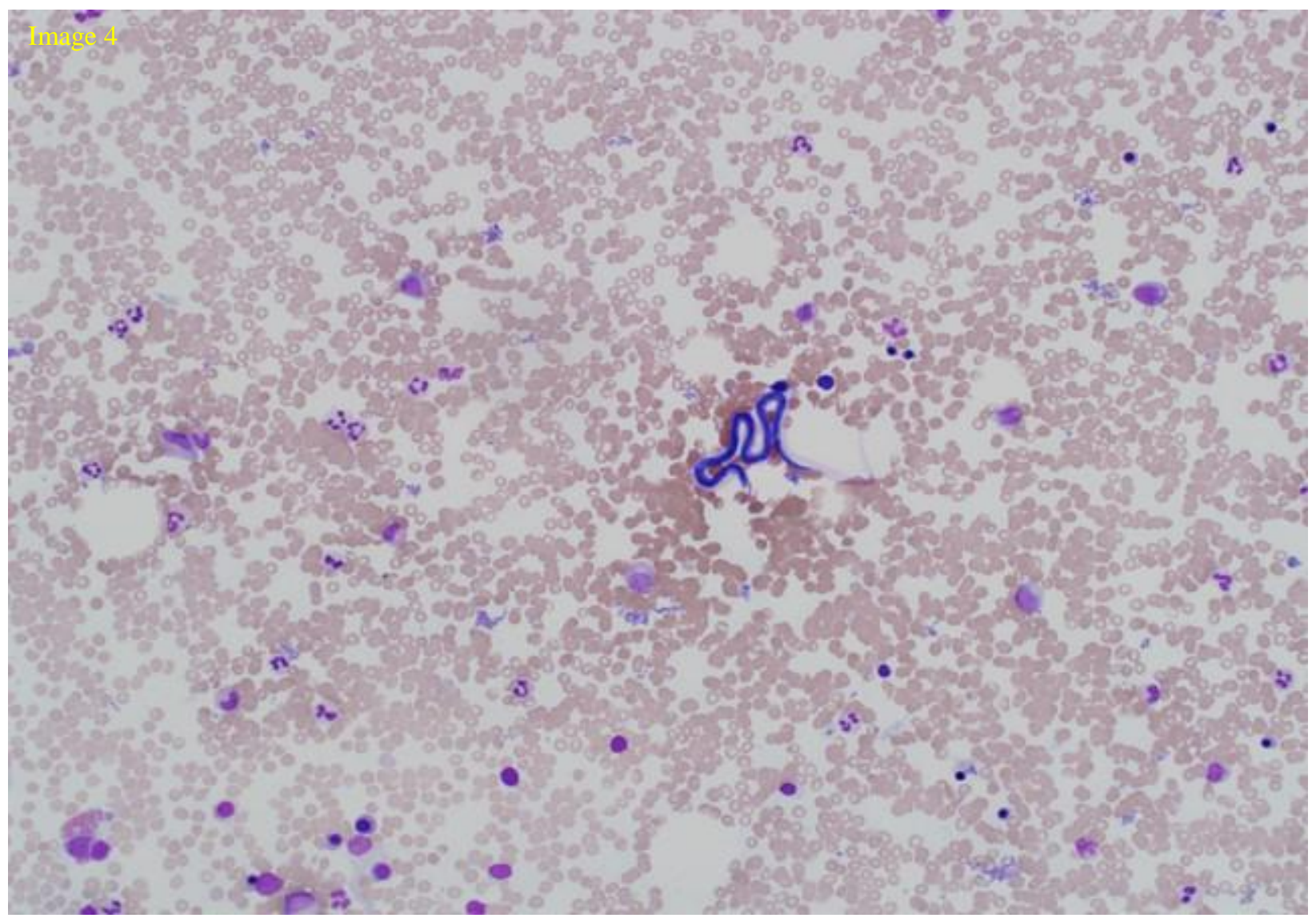




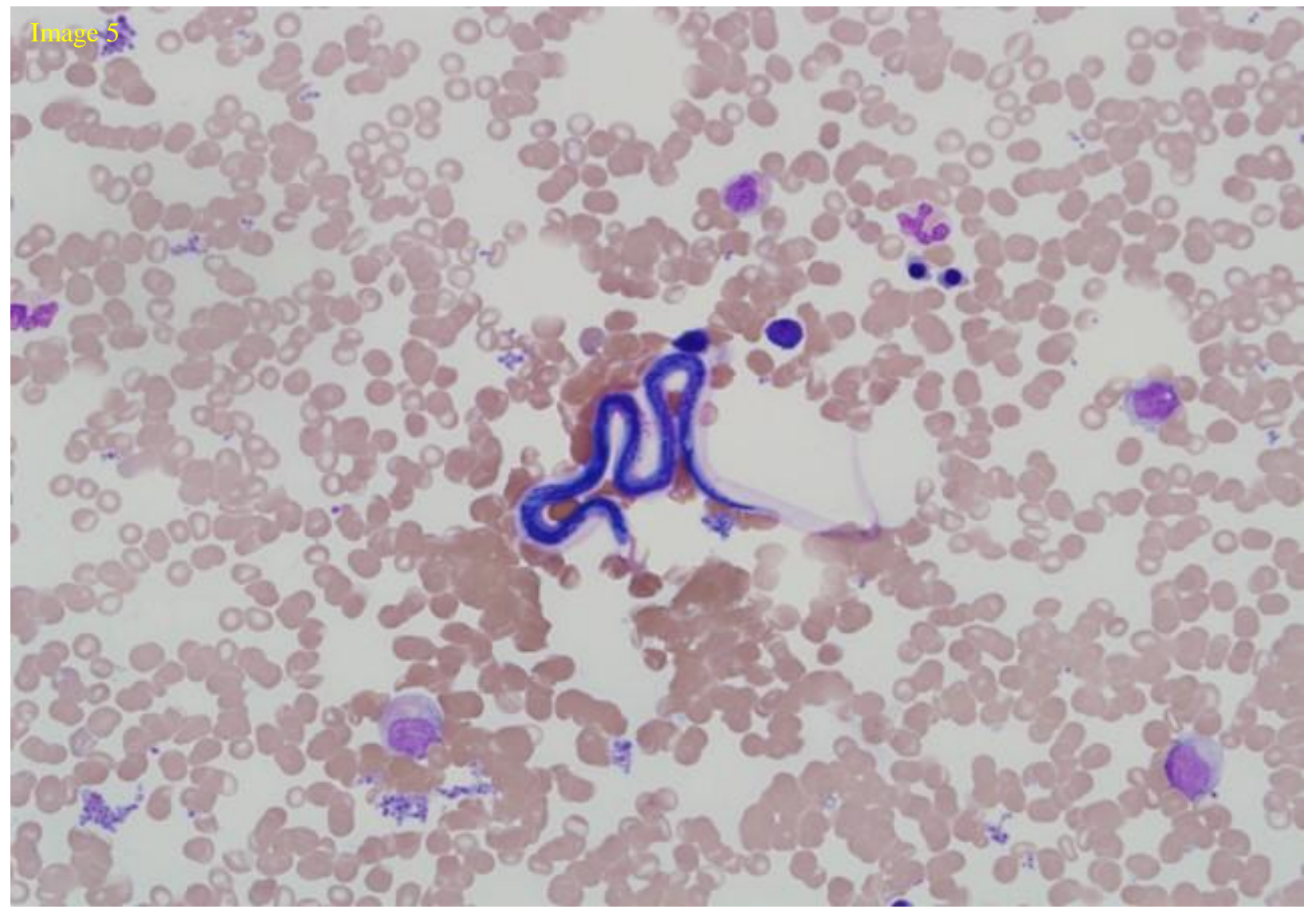

\section{Discussion:-}

Pradhan S reported that microfilariae of $W$. bancrofti were detected in seven cases of anemia in persons with asymptomatic Filariasis. In three cases microfilariae could not be detected in the blood. Their presence in bone marrow may indicate the ability of microfilariae to cross the vessel wall (13).

Panja et al., (12) described a case of atypical filariasis who had meningo-encephalitis, proptosis, fever, anemia and joint pain without any clinical features of lymphatic filariasis. The bone marrow aspirates had shown microfilariae. The microfilaria of $\mathrm{W}$. bancrofti is the most common type of parasite that is demonstrated in the bone marrow (6), (7), (9), (11). One case of Mansonellaperstans microfilaria was described in the bone marrow of HIV positive patient who also had neutropenia and a hypoplastic marrow (8). It has not been clearly understood how these microfilariae reach the bone marrow. According to Simon et al., much of the recent data on filaria/mycobacteria coinfection has focused on the modulation of mycobacteria-specific or malaria-specific responses by chronic filarial infection. As such, filarial infections very clearly alter the magnitude and quality of the mycobacteria-specific or malaria-specific cytokine responses, responses that have been typically associated with control of these intracellular pathogens (11). Peripheral blood eosinophilia is a common hematological finding in filariasis. But in a majority of the reported cases, in which microfilariae were demonstrated in the bone marrow, eosinophilia was absent (5), (7), (9). A similar finding was noted in our case also. The absence of peripheral blood eosinophilia in these cases may be attributed to the oxidative stress which was associated with the chronic and occult filariasis, which had caused altered immune responses (12). Anemia and pancytopenia were the other frequent peripheral blood findings (5), (6), (7), (9). The bone marrow may be aplastic, hypoplastic or hyperplastic with a normoblastic or a megaloblastic maturation (5), (7), (9), (11). The bone marrow hypoplasia may be attributed to drugs (5) and viral infections such as those which are caused by varicella (6) and HIV (8) or it may be idiopathic (5). Sharma et al., (5) described six cases of microfilariae in the bone marrow aspirates, among which five had bone marrow hypoplasia or aplastic. In two cases with aplastic anemia, the etiology was not known and it was considered as idiopathic. Shenoi et al., (9) described three cases of microfilariae in the bone marrow aspirates, among which one had aplastic anemia and the 
other had megakaryocytic aplasia. They opined that the blood and the bone marrow changes were unrelated to the filarial infestation. Arundhati et al., (10) described a case of acute lymphoblastic leukemia in association with W.bancrofti microfilariae in the bone marrow aspirates and it was likely that the immunocompromised status in the leukemia had made the patient more susceptible for the parasitic infestation. In our case, the pancytopenia, macrocytic RBCs and the hypersegmentation of the neutrophils were attributed to the megaloblastic anemia which was caused by a nutritional deficiency. We consider that the presence of microfilariae in the bone marrow is an incidental finding and that filariasis has no role in the causation of megaloblastic anemia. However, in the present case, the altered immune status which was associated with the anemia may be the cause of the asymptomatic microfilariae carrier status and the absence of the eosinophilia.

Pancytopenia with bone marrow infiltration by microfilariae has been rarely reported before. One case report previously showed microfilaria in the bone marrow aspirate of a young boy who presented with pancytopenia. The presentation in this boy was gum bleeding, but unlike our case. However no hemoparasite could be documented in the peripheral blood smear. Umashankar T, et al. reported another case of Filariasis in a 17 year old female where pancytopenia was the presenting feature. Though they documented microfilariae in marrow aspirate similar to our patient, there was evidence of megaloblastic anemia rather than marrow hypoplasia. In their opinion the presence of microfilaria in the marrow was an incidental finding. Presence of microfilaria in the marrow aspirate has also been supported by few other reports. Another case report by Tummidi et al described incidental finding of Microfilariae of $W$. bancrofti were incidentally detected in aspirate smears from a 65 year old lady while investigating back pain (14).Microfilariae circulate freely in the peripheral blood and may be trapped in various organs. They can be detected in the thyroid, breast, lymph nodes, subcutaneous nodules and in cervical scrape smears. This can explain the lodgment of microfilariae within bone marrow.

Absence of eosinophilia in our case may be due to altered immune status evoked by filariasis. The cause of marrow suppression in filariasis is uncertain. It has been postulated that it may be due to liberation of some toxic material by the growing microfilariae within the marrow. Mhimbira F et al. studied prevalence of helminth infections in 597 tuberculosis patients and 375 household controls, this study described finding of Strongyloides Stercoralis as the predominant helminth followed by hook worms and Schistosoma mansoni, W. bancrofti was not described as a finding (15). This case has been reported as an instance of rare presentation of Filariasis.

\section{Conclusions:-}

The important interactions between Mycobacterial infection and Filariasis must be considered. Their ability to cause inflammation and immunomodulation was demonstrated ex vivo, but whether this interplay affects disease outcome in either cases remains unknown.

Microfilariae in the bone marrow aspirates may be an incidental finding in the absence of the clinical features of filariasis. In the endemic areas, all the bone marrow aspirates must be screened for microfilariae to detect any asymptomatic carriers. Further documentation and investigations are needed to understand the possible etiological role of filariasis in various hematological manifestations such as pancytopenia and bone marrow hypoplasia.

\section{References:-}

1. Yenkeshwar PN, Kumbhalkar DT, Bobhate SK. Microfilariae in fine needle aspirates: a report of 22 cases. Indian J PatholMicrobiol 2006;49:365-69.

2. Kishore B, Khare P, Gupta RJ, Bisht SP. The microfilariae of Wuchereria bancrofti in cytologic smears: a report of 5 cases with unusual presentations. ActaCytol 2008;52:710-12.

3. Gupta S, Gupta R, Bansal B, Singh S, Gupta K, Kudesia M. The significance of the incidental detection of filariasis on aspiration smears: a case series. DiagnCytopathol 2010;38:517-20.

4. Anupindi L, Sahoo R, Rao RV, Verghese G, Rao PV. Microfilariae in the bronchial brushing cytology of symptomatic pulmonary lesions. A report of two cases. ActaCytol 1993;37:397-99.

5. Sharma S, Rawat A, Chowhan A. Microfilariae in bone marrow aspiration smears; their correlation with marrow hypoplasia: a report of six cases. Indian J PatholMicrobiol 2006;49:566-68.

6. Hemachandran M, Varma N, Varma S. Aplastic anaemia following a varicella infection with a coexistent microfilaraemia of Wuchereria bancrofti- a case report. Indian J PatholMicrobiol 2003;46:662-63.

7. Pradhan S, Lahiri VL, Elhence BR, Singh KN. The microfilariae of Wuchereria bancrofti in bone marrow smears. Am J Trop Med Hyg 1976;25:199-200. 
8. Molina MA, Cabezas MT, Giménez MJ. Mansonellaperstansfilariasis in an HIV patient: a finding in the bone marrow. Haematologica 1999;84:861.

9. Shenoi U, Pai RR, Pai U, Nandi GK, Adhikari P. Microfilariae in bone marrow aspiration smears. ActaCytol 1998;42:815-16.

10. Arundhati, Kumar A, Kumar R. Acute lymphoblastic leukaemia with microfilaria: a rare coincidence in bone marrow aspirates. Indian J Hematol Blood Transfus 2011; 27:111-12.

11. Simon Metenou, SubashBabu, Thomas B. Nutman. Impact of filarial infections on coincident intracellular pathogens: Mycobacterium tuberculosis and Plasmodium falciparum. CurrOpin HIV AIDS. 2012 May; 7(3): 231-238.

12. Panja M, Ganguly S, Kar AK, Chhetri MK. Atypical filariasis. J Assoc Physicians India 1987;35:596-97.

13. Pradhan S, Lahiri VL, Ethence BR, Singh KN. Micro-filariae of Wucheria bancrofti in bone marrow smear. Am J Trop Med Hyg 1976; 25(1):199-200.

14. TUMMIDI S, PATRO MK, BAL AK, CHOUDHURY A. MICROFILARIAE IN A BONE MARROW ASPIRATE. BMC RES NOTES. 2016 MAY 4;9:256.

15. Mhimbira F, Hella , Said K, Kamwela L, Sasamalo M, Maroa T, Chiryamkubi M, Mhalu G, Schindler C, Reither K, Knopp S, Utzinger J, Gagneux S, Fenner L. Prevalence and clinical relevance of helminth coinfections among tuberculosis patients in urban Tanzania. PLoSNegl Trop Dis. 2017 Feb 8;11(2):e0005342.

Figure Legends:-

Image1: Diffuse micro nodules bilaterally, reticulonodular changes in the apices with high suspicion of military TB Images 2 \& 3: Numerous miliary round ring enhancing lesions involving the supra and infratentorial parts of the brain, cerebellum and brainstem with localized perifocal edema and no significant mass effect. This is in conjunction with diffuse leptomeningeal and to lesser extent pachymeningeal enhancement, more concentrated at the basal cisterns and around brainstem with single lesion showing diffusion restriction in the left occipital lobe Images 4 \& 5: Light Microscopy picture of a bone marrow aspirate positive for W. bancrofti 